\title{
Topical Issue on Fluids and Structures: Multi-scale coupling and modeling
}

Published online: 12 March 2019 - C EDP Sciences / Società Italiana di Fisica / Springer-Verlag GmbH Germany, part of Springer Nature, 2019

Fluid-structure interaction is a broad topic of growing scientific interest, encompassing transdisciplinary contributions from basic and applied sciences with a range of potential applications. The latter include everyday life products and relevant processes from widespread industrial sectors, such as biomedical, food, household and personal care. Generally speaking, fluid-structure interaction is a challenging, multi-scale scientific problem where complexity arises from both the external flow field, such as in the case of turbulence, and the rheological behavior of the flowing matter, spanning from simple Newtonian fluids to viscoelastic multiphase systems with an underlying flow-dependent microstructure. In many cases, the multi-scale interplay between applied flows and complex fluids is still an open problem under active investigation.

This Topical Issue of The European Physical Journal E collects fifteen contributions at the crossroad of several disciplines, such as physics, bio-physics, applied mathematics and mechanical and chemical engineering. The contributions highlight research advances in diverse scientific problems related to flowing matter and structure interactions.

Several papers in the Topical Issue are addressed to multiphase systems with active or passive particles undergoing collective or individual motion. Examples of collective behavior of active soft matter include colloidal suspensions of swimming self-propelled particles by average metrics [1] and dense bacterial flows exhibiting a turbulence-like phase by a generalized Navier-Stokes equation [2]. Individual microswimmers are studied by a reinforcement learning approach to accomplish difficult motion tasks in complex fluid environments [3] and by solving the Stokes equations of the hydrodynamics in thin films [4]. Concerning other active particles, the motion of a spherical Janus particle near a no-slip wall [5] and the behavior of self-propelled liquid droplets under a Marangoni-like flow [6] are investigated by Lattice Boltzmann simulations. The flow behavior of passive droplets is studied both in concentrated emulsions by microscopy imaging of the flow-induced microstructure evolution [7] and under time-dependent flows using 3D numerical simulations of two immiscible fluids based on the Lattice Boltzmann model [8]. Turbulent flow with large neutrally buoyant particles in a pipe is the subject of a fully-resolved numerical simulation based on a Lattice Boltzmann scheme [9]. An inertial tracer advected by a two-dimensional incompressible laminar flow and subject to thermal noise is studied under the effect of an external force [10]. Drug release from a liposome into the bloodstream is tackled by two-dimensional simulations using the Lattice Boltzmann method and the immersed boundary method to handle the fluid-structure coupling [11]. Ventricular flow with a mitral valve is studied using direct numerical simulation of the Navier-Stokes equations and a comparison of the results with a companion dedicated experiment is also provided [12]. Some papers are devoted to nonlinear systems or turbulent flow of Newtonian fluids: these include energy transfer in turbulent shell models by using a reversible viscous mechanism [13], the dynamics of two-dimensional three-component flows in strongly rotating flows and/or of conducting fluids with a strong mean magnetic field [14], and the flow field surrounding cylindrical structures characterized by different types of corrugated surfaces [15].

The papers collected in this issue address the problem of fluid-structure interaction by different viewpoints based on contributions from basic and applied sciences. The diversity of approaches, from statistical mechanics to energy landscape analysis, provides a multi-scale picture of fluids and structures and outlines an active research area where new and emerging challenges point to exciting perspectives of further cross-fertilization among disciplines for future developments. 
We acknowledge support from COST Action MP1305, the European Research Council under the EU Seventh Framework Programme (FP7/2007-2013)/ ERC Grant Agreements no. 279004 and no. 339032. Some of the contributing authors participated in the conference Fluids and Structures: Interaction and Modeling, held in Napoli (Italy) from 22 to 26 May 2017, which inspired this Topical Issue.

Luca Biferale, Stefano Guido, Andrea Scagliarini and Federico Toschi Guest Editors

\section{References}

1. Raffaele Pastore, Marco Uttieri, Giuseppe Bianco, Maurizio Ribera d'Alcalá, Maria Grazia Mazzocchi, Eur. Phys. J. E 41, 79 (2018).

2. Martin James, Michael Wilczek, Eur. Phys. J. E 41, 21 (2018).

3. K. Gustavsson, L. Biferale, A. Celani, S. Colabrese, Eur. Phys. J. E 40, 110 (2017).

4. Daniela Pimponi, Mauro Chinappi, Paolo Gualtieri, Eur. Phys. J. E 41, 28 (2018).

5. Zaiyi Shen, Alois Würger, Juho S. Lintuvuori, Eur. Phys. J. E 41, 39 (2018).

6. F. Fadda, G. Gonnella, A. Lamura, A. Tiribocchi, Eur. Phys. J. E 40, 112 (2017).

7. Valentina Preziosi, Antonio Perazzo, Giovanna Tomaiuolo, Stefano Guido, Eur. Phys. J. E 41, 45 (2018).

8. F. Milan, M. Sbragaglia, L. Biferale, F. Toschi, Eur. Phys. J. E 41, 6 (2018).

9. A. Gupta, H.J.H. Clercx, F. Toschi, Eur. Phys. J. E 41, 34 (2018).

10. F. Cecconi, A. Puglisi, A. Sarracino, A. Vulpiani, Eur. Phys. J. E 40, 81 (2017).

11. Badr Kaoui, Eur. Phys. J. E 41, 20 (2018).

12. Valentina Meschini, Marco D. de Tullio, Roberto Verzicco, Eur. Phys. J. E 41, 27 (2018).

13. Massimo De Pietro, Luca Biferale, Guido Boffetta, Massimo Cencini, Eur. Phys. J. E 41, 48 (2018).

14. Moritz Linkmann, Michele Buzzicotti, Luca Biferale, Eur. Phys. J. E 41, 4 (2018).

15. Vesselin K. Krastev, Giorgio Amati, Sauro Succi, Giacomo Falcucci, Eur. Phys. J. E 41, 95 (2018). 